TRANSACTIONS OF THE

AMERICAN MATHEMATICAL SOCIETY

Volume 357, Number 8, Pages 3339-3358

S 0002-9947(05)03699-8

Article electronically published on March 10, 2005

\title{
TELESCOPING, RATIONAL-VALUED SERIES, AND ZETA FUNCTIONS
}

\author{
J. MARSHALL ASH AND STEFAN CATOIU
}

\begin{abstract}
We give an effective procedure for determining whether or not a series $\sum_{n=M}^{N} r(n)$ telescopes when $r(n)$ is a rational function with complex coefficients. We give new examples of series $(*) \sum_{n=1}^{\infty} r(n)$, where $r(n)$ is a rational function with integer coefficients, that add up to a rational number. Generalizations of the Euler phi function and the Riemann zeta function are involved. We give an effective procedure for determining which numbers of the form $(*)$ are rational. This procedure is conditional on 3 conjectures, which are shown to be equivalent to conjectures involving the linear independence over the rationals of certain sets of real numbers. For example, one of the conjectures is shown to be equivalent to the well-known conjecture that the set $\{\zeta(s): s=2,3,4, \ldots\}$ is linearly independent, where $\zeta(s)=\sum n^{-s}$ is the Riemann zeta function.

Some series of the form $\sum_{n} s(\sqrt[r]{n}, \sqrt[r]{n+1}, \cdots, \sqrt[r]{n+k})$, where $s$ is a quotient of symmetric polynomials, are shown to be telescoping, as is $\sum 1 /(n !+(n-1) !)$. Quantum versions of these examples are also given.
\end{abstract}

\section{INTRODUCTION}

The first example of telescoping that one usually meets is the sum

$$
\sum_{n=1}^{\infty} \frac{1}{n(n+1)}=\sum_{n=1}^{\infty} \frac{1}{n}-\frac{1}{n+1}=1 .
$$

This is just the tip of a very big iceberg. First of all, given any convergent sequence $\left\{s_{n}\right\}$, we can display its limit as the telescoping series

$$
s_{1}-\sum_{n=1}^{\infty}\left(s_{n}-s_{n+1}\right) \text {. }
$$

A general class of sums where telescoping is often used are sums of the form

$$
\sum_{n=1}^{N} p(n)
$$

Received by the editors August 12, 2003 and, in revised form, February 21, 2004.

2000 Mathematics Subject Classification. Primary 11J72, 11M41, 11A25, 40 A25.

Key words and phrases. Generalized zeta function, generalized Euler phi function, linear independence over the rationals.

The first author's research was partially supported by NSF grant DMS 9707011 and a grant from the Faculty and Development Program of the College of Liberal Arts and Sciences, DePaul University.

(C)2005 American Mathematical Society Reverts to public domain 28 years from publication 
where $p$ is a polynomial. Write $p$ using the basis $\{1, x, x(x-1), x(x-1)(x-2), \ldots\}$ and let $P$ be the discrete "integral" of $p$ defined on the basis by the operator $1 \mapsto-x, x(x-1) \cdots(x-(k-1)) \mapsto \frac{-1}{k+1} x(x-1) \cdots(x-k), k=1,2, \cdots$, and extended to be linear. For example, if $p(n)=5+7 n+11 n(n-1)$, then $P(n)=$ $-5 n-7 \cdot \frac{1}{2} n(n-1)-11 \cdot \frac{1}{3} n(n-1)(n-2)$. Then $P(n)-P(n+1)=p(n)$ and we may evaluate the original sum as

$$
\sum_{n=1}^{N} p(n)=\sum_{n=1}^{N}(P(n)-P(n+1))=P(1)-P(N+1) .
$$

(See Chapters 22-24 of [KC], wherein telescoping is viewed as a discrete version of the fundamental theorem of calculus.)

To appreciate this integration process, first notice that one can always form a discrete integral. For if $\sum_{n=1}^{\infty} a_{n}$ is any convergent series, define $s_{0}=0$, and for any $n \geq 1$, let $s_{n}=a_{1}+a_{2}+\cdots+a_{n}$. Then $s_{n}-s_{n-1}=a_{n}$ and $\sum_{n=1}^{N} a_{n}=$ $\sum_{n=1}^{N} s_{n}-s_{n-1}=s_{N}$. What is the difference between the useful construction of $P(n)$ from $p(n)$ and the formal and not very helpful construction of $s_{n}$ from the sequence of $a_{i}$ 's is that $P(n)$ is given by a simple formula whose length is independent of $n$.

For a slight generalization of the original example, let us look at rational expressions which can be written in the form $R(n)-R(n+d)$, where $d$ is a positive integer greater than 1 . These can also be telescoped, but one needs to operate $d$ telescopes simultaneously. For example,

$$
\begin{aligned}
\sum_{n=1}^{\infty} \frac{1}{n}-\frac{1}{n+3} & =\sum_{n=1}^{\infty} \frac{1}{n}-\frac{1}{n+1}+\frac{1}{n+1}-\frac{1}{n+2}+\frac{1}{n+2}-\frac{1}{n+3} \\
& =\sum_{n=1}^{\infty}\left(\frac{1}{n}+\frac{1}{n+1}+\frac{1}{n+2}\right)-\left(\frac{1}{n+1}+\frac{1}{n+2}+\frac{1}{n+3}\right) \\
& =1+\frac{1}{2}+\frac{1}{3} .
\end{aligned}
$$

It is natural to ask which rational functions of $n$ have discrete integrals that are also rational functions of $n$ ? For example, we know that $d(n)=\frac{1}{n(n+1)}$ has discrete integral $D(n)=\frac{1}{n}$ in the sense that $D(n)-D(n+1)=d(n)$; what are the rational functions that are expressible as linear combinations of differences of rational functions? More explicitly, say that the rational function over the integers $r(n)$ is a rational differential if there is a rational function over the integers $R$ so that $R(n)-R(n+1)=r(n)$. So $\sum r(n)$ telescopes, by definition, exactly when $r(n)$ is a rational differential. Our first result is an effective procedure for determining when a given rational function over the integers $r(n)$ is a rational differential. Write $r(n)$ in partial fraction form, $r(n)=q(n)+\sum_{(f, s) \in S} A_{f, s}(n) / f(n)^{s}$. Here $S$ is a finite subset of $\mathbb{Q}[n] \times \mathbb{N}$, the product of the polynomials with rational coefficients and the natural numbers; $A_{f, s} \in \mathbb{Q}[n]$ with $\operatorname{deg} A_{f, s}<\operatorname{deg} f$; and each $f$ is monic and irreducible in $\mathbb{Q}[n]$. As we mentioned before, $q$ is a rational differential, so we only need to determine which sums of the form

$$
\sum_{(f, s) \in S} \frac{A_{f, s}(n)}{f(n)^{s}}
$$


are rational differentials. Given such a sum, partition $S$ into components using the equivalence relation $(f, s) \sim(g, t)$ if there is an integer $k$ such that $g(n)=f(n+k)$ for all $n$ and $s=t$. This induces a decomposition of the sum into a sum of sub-sums. For example,

$$
\left\{\frac{3+n}{\left(n+\frac{7}{2}\right)^{2}+3}+\frac{4+2(n-1)}{\left(n+\frac{5}{2}\right)^{2}+3}+\frac{A+B(n-4)}{\left(n-\frac{1}{2}\right)^{2}+3}\right\}+\left\{\frac{6}{\left(n+\frac{1}{3}\right)^{2}}+\frac{C}{\left(n+\frac{7}{3}\right)^{2}}\right\}+\left\{\frac{D}{(n+\pi i)^{7}}\right\}
$$

is a partial fraction sum that decomposes into three sub-sums, each enclosed by a pair of braces. The necessary and sufficient condition for being a rational differential is that the coefficients of each sub-sum add to zero. In particular this means that our example is a rational differential exactly when $(A, B, C, D)=(-7,-3,-6,0)$ so that $(3+n)+(4+2 n)+(A+B n)=0,6+C=0$, and $D=0$. See Section 2 and especially Theorem 1 for the general statement and proof.

Consider the question of when $\sum_{n=1}^{\infty} r(n)$, where $r(n)$ is a rational function of $n$ with integer coefficients, sums up to a rational number $s$. We know that $s$ is rational if $r(n)$ is a rational differential over $\mathbb{Q}(n)$. If the denominator of $r$ factors into linear factors, we subtract an appropriate telescoping series from $\sum r(n)$ to obtain a simpler series, called its standard form, which has the form

$$
\sum_{s} \sum_{\substack{\alpha \in \mathbb{Q} \\ \alpha \in(0,1]}} \frac{A_{\alpha s}}{(n+\alpha)^{s}}
$$

For example, if $r(n)$ is given by

$$
\left\{\frac{3}{\left(n+\frac{7}{2}\right)^{2}}+\frac{2}{\left(n+\frac{5}{2}\right)^{2}}+\frac{A}{\left(n-\frac{1}{2}\right)^{2}}\right\}+\left\{\frac{6}{\left(n+\frac{1}{3}\right)^{2}}+\frac{B}{\left(n+\frac{7}{3}\right)^{2}}\right\}+\left\{\frac{C}{n^{7}}\right\}
$$

then the associated standard form is

$$
\left\{\frac{3+2+A}{\left(n+\frac{1}{2}\right)^{2}}\right\}+\left\{\frac{6+B}{\left(n+\frac{1}{3}\right)^{2}}\right\}+\left\{\frac{C}{(n+1)^{7}}\right\} .
$$

When the denominator of $r$ factors into linear factors, the rationality of a series is equivalent to the rationality of its standard form, and a series telescopes exactly when its standard form is zero. Here is a way to get a rational sum from a standard form. Start with three different decompositions of $\zeta(2)=\sum n^{-2}=\pi^{2} / 6$ :

$$
\begin{aligned}
& \sum_{n=1}^{\infty} \frac{1}{(2 n)^{2}}+\sum_{n=0}^{\infty} \frac{1}{(2 n+1)^{2}}=\frac{1}{4}\left(\sum_{n=1}^{\infty} \frac{1}{n^{2}}+\sum_{n=0}^{\infty} \frac{1}{(n+1 / 2)^{2}}\right), \\
& \frac{1}{16}\left(\sum_{n=1}^{\infty} \frac{1}{n^{2}}+\sum_{n=0}^{\infty} \frac{1}{(n+1 / 4)^{2}}+\sum_{n=0}^{\infty} \frac{1}{(n+2 / 4)^{2}}+\sum_{n=0}^{\infty} \frac{1}{(n+3 / 4)^{2}}\right) \text {, and } \\
& \frac{1}{64}\left(\begin{array}{c}
\sum_{n=1}^{\infty} \frac{1}{n^{2}}+\sum_{n=0}^{\infty} \frac{1}{(n+1 / 8)^{2}}+\sum_{n=0}^{\infty} \frac{1}{(n+2 / 8)^{2}}+\sum_{n=0}^{\infty} \frac{1}{(n+3 / 8)^{2}} \\
+\sum_{n=0}^{\infty} \frac{1}{(n+4 / 8)^{2}}+\sum_{n=0}^{\infty} \frac{1}{(n+5 / 8)^{2}}+\sum_{n=0}^{\infty} \frac{1}{(n+6 / 8)^{2}}+\sum_{n=0}^{\infty} \frac{1}{(n+7 / 8)^{2}}
\end{array}\right) .
\end{aligned}
$$

Let $\zeta_{2}(2)=\sum_{n=0}^{\infty} \frac{1}{(n+1 / 2)^{2}}, \zeta_{4}(2)=\sum_{n=0}^{\infty} \frac{1}{(n+1 / 4)^{2}}+\sum_{n=0}^{\infty} \frac{1}{(n+3 / 4)^{2}}$ and $\zeta_{8}(2)=$ $\sum_{n=0}^{\infty} \frac{1}{(n+1 / 8)^{2}}+\sum_{n=0}^{\infty} \frac{1}{(n+3 / 8)^{2}}+\sum_{n=0}^{\infty} \frac{1}{(n+5 / 8)^{2}}+\sum_{n=0}^{\infty} \frac{1}{(n+7 / 8)^{2}}$. The above three 
representations for $\zeta(2)$ can easily be solved for $\zeta_{2}, \zeta_{4}$, and $\zeta_{8}$, giving

$$
\zeta_{2}=3 \zeta(2), \zeta_{4}=12 \zeta(2) \text {, and } \zeta_{8}=48 \zeta(2) \text {. }
$$

These identities lead to many previously unknown infinite series with rational sums. If $(a, b, c) \in \mathbb{Q}^{3}$ is such that $3 a+12 b+48 c=0$, then $a \zeta_{2}+b \zeta_{4}+c \zeta_{8}=0 \in \mathbb{Q}$. Similarly, $\sum_{n=1}^{\infty} 3 \frac{1}{n^{2}}-\frac{1}{(n+3 / 2)^{2}}=3 \zeta(2)-\zeta_{2}+\frac{1}{(1 / 2)^{2}}+\frac{1}{(3 / 2)^{2}}=\frac{40}{9}$. By Corollary 1 neither of these sums is rationally telescoping. We conjecture that the examples presented so far demonstrate the only ways that a rational sum can be achieved. We will make this conjecture explicit in Section 4 below. In that section we will give equivalent conjectures which seem plausible. The equivalent conjectures are extensions of the well-known fact that the set $\{1, \zeta(2), \zeta(4), \zeta(6), \ldots\}$ is linearly independent over $\mathbb{Q}$. In particular, if some "atom" such as $\zeta(5)=\sum n^{-5}$ turned out to be rational, this whole classification conjecture would fall apart.

In Section [5] we give a few simple examples that were the original motivation for writing this paper and then extend the notion of telescoping (which corresponds to first differences) to that of $k$-telescoping (which corresponds to $k$ th differences). The examples in this section lead to two interesting formulas involving binomial coefficients, formulas (5.3) and (5.4). The proofs that we found for them are short and perhaps unexpected. The remainder of this paper gives two other kinds of examples of telescoping beyond rational functions. The first involves generalizations of this example involving square roots:

$$
\begin{aligned}
\sum_{n=1}^{N} \frac{1}{\sqrt{n}+\sqrt{n+1}} & =\sum_{n=1}^{N} \frac{1}{\sqrt{n+1}+\sqrt{n}} \frac{\sqrt{n+1}-\sqrt{n}}{\sqrt{n+1}-\sqrt{n}} \\
& =\sum_{n=1}^{N}-\sqrt{n}+\sqrt{n+1}=\sqrt{N+1}-1 .
\end{aligned}
$$

The computational details are straightforward, and hence they are omitted. The second example involves factorials:

$$
\sum_{n=1}^{\infty} \frac{1}{(n-1) !+n !}=\sum_{n=1}^{\infty}\left(\frac{1}{n !}-\frac{1}{(n+1) !}\right)=1 .
$$

We also investigate quantum versions of all these examples.

The authors are happy to provide unpublished proofs for all of the results in Sections 6 and 7 as well as computational evidence for Conjecture 2.

\section{Characterization of telescoping}

The main object of our investigation in this paper is convergent series of rational functions with coefficients in the field $\mathbb{Q}$ of rational numbers. By the well-known Limit Comparison Test and the $p$-Test, these are series of the form $\sum_{n=1}^{\infty} P(n) / Q(n)$, where $P, Q \in \mathbb{Q}[x]$ satisfy the condition $\operatorname{deg} Q \geq \operatorname{deg} P+2$. For this reason, we will restrict our attention to the set $\mathbb{Q}^{-}(x)=\{P(x) / Q(x): P, Q \in \mathbb{Q}[x], Q$ monic, $\operatorname{deg} P<\operatorname{deg} Q\}$ of all subunitary rational functions over $\mathbb{Q}$. This is a $\mathbb{Q}$-subspace of the field $\mathbb{Q}(x)$ of all rational functions over $\mathbb{Q}$. In fact, via long division and as a $\mathbb{Q}$-vector space, $\mathbb{Q}(x)$ is the direct sum $\mathbb{Q}(x)=\mathbb{Q}[x] \oplus \mathbb{Q}^{-}(x)$.

If $r(x) \in \mathbb{Q}(x)$ is a rational function over $\mathbb{Q}$, we say that the series $\sum_{n=1}^{\infty} r(n)$ telescopes over $\mathbb{Q}$ if its $n$th partial sum $s_{n}=\sum_{k=1}^{n} r(k)$ is a rational function of $n$ over $\mathbb{Q}$. This is equivalent to saying that $r(n)$ is a rational differential over $\mathbb{Q}$, 
i.e., $r(n)=R(n)-R(n+1)$ for some rational function $R(x) \in \mathbb{Q}(x)$. In fact $R(n)=-s_{n-1}+R(1)$, for $n>1$.

Let $P / Q$ be an element of $\mathbb{Q}^{-}(x)$. If $Q=f_{1}^{s_{1}} f_{2}^{s_{2}} \ldots f_{t}^{s_{t}}$ is the prime factorization of $Q$ in $\mathbb{Q}[x]$, with $f_{i}$ 's being distinct monic irreducible polynomials over $\mathbb{Q}$, then by the well-known partial simple fractions decomposition theorem, we can write $P / Q$ uniquely as a sum

$$
\frac{P(x)}{Q(x)}=\sum_{i=1}^{t} \sum_{j=1}^{s_{i}} \frac{A_{i, j}(x)}{f_{i}(x)^{j}}
$$

where $A_{i, j} \in \mathbb{Q}[x]$ satisfy $\operatorname{deg} A_{i, j}<\operatorname{deg} f_{i}$, for all $i, j$. Moreover, without losing uniqueness, we can extend this expression by adding infinitely many zero terms so that $P / Q$ can be written uniquely as an infinite sum of finite support:

$$
\frac{P(x)}{Q(x)}=\sum_{\substack{f \in \mathbb{Q}[x] \\ f \text { monic irreducible }}} \sum_{s>0} \frac{A_{f, s}(x)}{f^{s}(x)},
$$

where the $A_{f, s} \in \mathbb{Q}[x]$ and satisfy $\operatorname{deg} A_{f, s}<\operatorname{deg} f$, for all $f, s$. Furthermore, if $f$ and $g$ are monic irreducible polynomials in $\mathbb{Q}[x]$, we say that $f \sim g$ if there is an integer $k$ such that $g(x)=f(x+k)$. This is an equivalence relation on the set of all monic irreducible polynomials in $\mathbb{Q}[x]$. Let $\mathcal{R}$ be a complete system of representatives of the equivalence classes for this relation. Later on we will make the assumption that the degree 1 elements of $\mathcal{R}$ are of the form $x+\alpha$, with $\alpha \in(0,1] \cap \mathbb{Q}$.

Then $P / Q$ can be written uniquely as an infinite sum of finite support:

$$
\frac{P(x)}{Q(x)}=\sum_{f \in \mathcal{R}} \sum_{s>0} \sum_{r \in \mathbb{Z}} \frac{A_{f, s, r}(x+r)}{f^{s}(x+r)}
$$

where $A_{f, s, r} \in \mathbb{Q}[x]$ satisfy $\operatorname{deg} A_{f, s, r}<\operatorname{deg} f$, for all $f, s, r$. This is equivalent to saying that the set

$$
\mathcal{B}=\bigcup_{f \in \mathcal{R}}\left\{\frac{(x+r)^{m}}{f^{s}(x+r)}: r, s, m \in \mathbb{Z}, s>0,0 \leq m<\operatorname{deg} f\right\}
$$

is a $\mathbb{Q}$-basis of $\mathbb{Q}^{-}(x)$.

The difference operator $\Delta: \mathbb{Q}(x) \longrightarrow \mathbb{Q}(x)$, defined by $(\Delta r)(x)=r(x)-r(x+1)$ for all $r \in \mathbb{Q}(x)$, is clearly $\mathbb{Q}$-linear. Its image $\Delta \mathbb{Q}(x)$ is the $\mathbb{Q}$-space of all rational differentials over $\mathbb{Q}$. We observe that both $\mathbb{Q}[x]$ and $\mathbb{Q}^{-}(x)$ are invariant subspaces of $\Delta$, so the subspace of all subunitary rational differentials over $\mathbb{Q}$ is $\Delta \mathbb{Q}(x) \cap$ $\mathbb{Q}^{-}(x)=\Delta \mathbb{Q}^{-}(x)$. In particular, $\Delta \mathbb{Q}^{-}(x)$ is spanned by the set

$$
\Delta \mathcal{B}=\bigcup_{f \in \mathcal{R}}\left\{\frac{(x+r+1)^{m}}{f^{s}(x+r+1)}-\frac{(x+r)^{m}}{f^{s}(x+r)}: r, s, m \in \mathbb{Z}, s>0,0 \leq m<\operatorname{deg} f\right\} .
$$

Moreover, since $\frac{(x+r+1)^{m}}{f^{s}(x+r+1)}-\frac{(x+r)^{m}}{f^{s}(x+r)}=\left(\frac{(x+r+1)^{m}}{f^{s}(x+r+1)}-\frac{x^{m}}{f^{s}(x)}\right)-\left(\frac{(x+r)^{m}}{f^{s}(x+r)}-\frac{x^{m}}{f^{s}(x)}\right)$, the set $\Delta \mathcal{B}$ and therefore $\Delta \mathbb{Q}^{-}(x)$ is spanned by the set

$$
\mathcal{B}_{0}=\bigcup_{f \in \mathcal{R}}\left\{\frac{(x+r)^{m}}{f^{s}(x+r)}-\frac{x^{m}}{f^{s}(x)}: r, s, m \in \mathbb{Z}, r \neq 0, s>0,0 \leq m<\operatorname{deg} f\right\} .
$$

By taking linear combinations and putting together the terms with the same denominator, what we have just proved is that every subunitary rational differential 
$P / Q$ can be written in the form

$$
\frac{P(x)}{Q(x)}=\sum_{f \in \mathcal{R}} \sum_{s>0} \sum_{r \in \mathbb{Z} \backslash\{0\}}\left(\frac{A_{f, s, r}(x+r)}{f^{s}(x+r)}-\frac{A_{f, s, r}(x)}{f^{s}(x)}\right),
$$

where $A_{f, s, r} \in \mathbb{Q}[x]$ satisfy $\operatorname{deg} A_{f, s, r}<\operatorname{deg} f$, for all $f, s, r$.

How can we tell whether a specific rational function such as $s(x)=\frac{1}{x^{2}}$ is a rational differential? Of course, those of us who know that $\sum_{n=1}^{\infty} \frac{1}{n^{2}}=\frac{\pi^{2}}{6}$ have a pretty strong suspicion that in this case the answer is no. A very general answer to the question is given by the following classification theorem, which gives a necessary and sufficient condition for a rational function to be a rational differential.

Theorem 1. Let $P, Q \in \mathbb{Q}[x]$ be polynomials with rational coefficients such that $\operatorname{deg} P<\operatorname{deg} Q$, and let $\mathcal{R}$ be a complete system of representatives of monic irreducible polynomials in $\mathbb{Q}[x]$ via the equivalence relation $\sim$ defined above. Then:

(i) The form $P / Q \in \mathbb{Q}(x)$ can be expressed uniquely as

$$
\frac{P(x)}{Q(x)}=\sum_{f \in \mathcal{R}} \sum_{s>0} \sum_{r \in \mathbb{Z}} \frac{A_{f, s, r}(x+r)}{f^{s}(x+r)},
$$

where the $A_{f, s, r} \in \mathbb{Q}[x]$ and satisfy $\operatorname{deg} A_{f, s, r}<\operatorname{deg} f$ for all $f, s, r$.

(ii) The rewriting

$$
\frac{P(x)}{Q(x)}=\sum_{f \in \mathcal{R}} \sum_{s>0} \sum_{r \in \mathbb{Z} \backslash\{0\}}\left(\frac{A_{f, s, r}(x+r)}{f^{s}(x+r)}-\frac{A_{f, s, r}(x)}{f^{s}(x)}\right)+\sum_{f \in \mathcal{R}} \sum_{s>0} \frac{\sum_{r \in \mathbb{Z}} A_{f, s, r}(x)}{f^{s}(x)}
$$

of the expression in part (i) is a unique decomposition of $P / Q$ as a sum of a rational differential form and a non-rational differential form over $\mathbb{Q}$. These are the "rational differential part" and the "representative part" of $P / Q$.

(iii) The series $\sum_{n=1}^{\infty} P(n) / Q(n)$ telescopes over $\mathbb{Q}$ if and only if

$P / Q$ is a rational differential over $\mathbb{Q}$ if and only if

the representative part of $P / Q$ is zero if and only if

$\sum_{r \in \mathbb{Z}} A_{f, s, r}(x)=0$, for all $f$ and $s$.

Proof. Part (i) is the standard partial fraction representation. We have already shown that the first sum in part (ii) is a rational differential. It remains to prove both uniqueness and that the second sum is not a rational differential. It suffices to show, for both, that the only way the second sum is a rational differential is when it is zero. Indeed, if the representative part of $P / Q$ is a rational differential, then, by the comments preceding the theorem, we can write it as

$$
\sum_{f \in \mathcal{R}} \sum_{s>0} \frac{\sum_{r \in \mathbb{Z}} A_{f, s, r}(x)}{f^{s}(x)}=\sum_{f \in \mathcal{R}} \sum_{s>0} \sum_{r \in \mathbb{Z} \backslash\{0\}}\left(\frac{B_{f, s, r}(x+r)}{f^{s}(x+r)}-\frac{B_{f, s, r}(x)}{f^{s}(x)}\right),
$$

where the $B_{f, s, r} \in \mathbb{Q}[x]$ and they satisfy $\operatorname{deg} B_{f, s, r}<\operatorname{deg} f$ for all $f, s, r$. Since $f(x+r) \notin \mathcal{R}$, for all $f \in \mathcal{R}$ and $r \neq 0$, uniqueness in part (i) implies that $B_{f, s, r}(x+r)=0$, for all $s$. Replacing $x$ by $x-r$ gives $B_{f, s, r}(x)=0$, for all $f, s, r$. Thus the right sum is zero and this finishes the proof of part (ii). The first equivalence in part (iii) comes by definition, the second comes from the uniqueness in part (ii), and the third comes from the uniqueness in part (i).

An interesting particular case of this theorem is when $Q$ splits, i.e., factors completely over $\mathbb{Q}$. 
Corollary 1. Let $P(x) / Q(x)$ be a rational function over $\mathbb{Q}$, and suppose that $Q$ splits over $\mathbb{Q}$. Then $P / Q$ is a rational differential over $\mathbb{Q}$ (or, equivalently, the series $\sum_{n=1}^{\infty} P(n) / Q(n)$ telescopes over $\left.\mathbb{Q}\right)$ if and only if $P / Q$ has a unique expression as an infinite sum of finite support

$$
\frac{P(x)}{Q(x)}=\sum_{s=1}^{\infty} \sum_{\mathcal{C} \in \mathbb{Q} / \mathbb{Z}} \sum_{\alpha \in \mathcal{C}} \frac{A_{\alpha, s}}{(x-\alpha)^{s}},
$$

where for every positive integer $s$ and every coset $\mathcal{C}$ of $\mathbb{Q} / \mathbb{Z}$,

$$
\sum_{\alpha \in \mathcal{C}} A_{\alpha, s}=0 .
$$

Here $Q(x) \in \mathbb{Q}[x]$ and $A_{\alpha, s} \in \mathbb{Q}$, for all $\alpha \in \mathbb{Q}$, and integers $s>0$.

This corollary is illustrated by the passage from (1.4) to (1.5) in the Introduction.

\section{EULER $\varphi$-FUNCTIONS AND ZETA FUNCTIONS}

We begin by introducing a generalization of the Euler phi-function. For each positive integer $s$, let $\varphi_{s}(a)$ be defined as the cardinality of the set

$$
\Phi_{s}(a)=\left\{m \in Z: 1 \leq m \leq a^{s} \text { and for every prime } p, p^{s} \nmid \operatorname{gcd}\left(m, a^{s}\right)\right\} .
$$

Note that $\varphi_{1}$ is the usual Euler phi-function. Then $\varphi_{s}$ has similar properties to $\varphi_{1}$, some of which are listed in the next theorem.

Theorem 2. The function $\varphi_{s}$ enjoys the following properties:

(1) The function $\varphi_{s}$ is multiplicative, $\varphi_{s}(a b)=\varphi_{s}(a) \varphi_{s}(b)$ for $\operatorname{gcd}(a, b)=1$.

(2) If $p$ is a prime number, then $\varphi_{s}\left(p^{\ell}\right)=p^{s \ell}-p^{s(\ell-1)}$.

(3) For any positive integer $a=p_{1}^{e_{1}} p_{2}^{e_{2}} \cdots p_{t}^{e_{t}}$,

$$
\varphi_{s}(a)=a^{s}\left(1-\frac{1}{p_{1}^{s}}\right)\left(1-\frac{1}{p_{2}^{s}}\right) \cdots\left(1-\frac{1}{p_{t}^{s}}\right) .
$$

(4) For every integer $a \geq 2$,

$$
a^{s}=\sum_{d \mid a} \varphi_{s}(d) .
$$

(5) For every integer $a \geq 2$,

$$
\varphi_{s}(a)=\sum_{d \mid a} \mu\left(\frac{a}{d}\right) d^{s},
$$

where $\mu$ is the Möbius function defined by

$$
\mu(n)=\left\{\begin{array}{cc}
1 & \text { if } n=1, \\
0 & \text { if } a^{2} \mid n \text { for some } a>1, \\
(-1)^{r} & \text { if a is a product of } r \text { distinct primes. }
\end{array}\right.
$$

(6) For every integer $a \geq 2$,

$$
\sum_{m \in \Phi_{s}(a)} m=\frac{1}{2} a^{s} \varphi_{s}(a)
$$


Proof. For property (1), find integers $\mu$ and $\nu$ so that $\mu a^{s}+\nu b^{s}=1$. For $(m, n) \in$ $\Phi_{s}(a) \times \Phi_{s}(b)$, let $k$ be the unique integer so that $x=n \mu a^{s}+m \nu b^{s}+k a^{s} b^{s} \in$ $\left[1, a^{s} b^{s}\right]$. Then show that the mapping $(m, n) \longmapsto x$ is a surjection from $\Phi_{s}(a) \times$ $\Phi_{s}(b)$ to $\Phi_{s}(a b)$. Property (2) is straightforward and property (3) is immediate from properties (1) and (2). Here and below $\operatorname{gcd}(-,-)$ will be abbreviated to $(-,-)$. For property (4), for each positive $d$ dividing $a$, define

$$
A_{d}=\left\{m \in\left[1, a^{s}\right]:\left(m, a^{s}\right)=d^{s} e, \text { with } p^{s} \nmid e \text { for every prime } p\right\} .
$$

The cardinality of $A_{d}$ is $\varphi_{s}(a / d)$ and the set $\left\{1,2, \ldots, a^{s}\right\}$ is partitioned by the family $\left\{A_{d}: d \mid a\right\}$, so $a^{s}=\sum_{d \mid a} \varphi_{s}(a / d)=\sum_{d \mid a} \varphi_{s}(d)$. Property (5) is a direct application of the Möbius Inversion Formula to equation (3.1) ([HW], p. 237). Let $\Phi_{s}(a)=\left\{a_{1}, a_{2}, \ldots, a_{\varphi_{s}(a)}\right\}$. Since $\left(m, a^{s}\right)=\left(a^{s}-m, a^{s}\right)$, we also have $\Phi_{s}(a)=$ $\left\{a^{s}-a_{1}, a^{s}-a_{2}, \ldots, a^{s}-a_{\varphi_{s}(a)}\right\}$. Thus $a_{1}+a_{2}+\cdots+a_{\varphi_{s}(a)}=a^{s}-a_{1}+a^{s}-$ $a_{2}+\cdots+a^{s}-a_{\varphi_{s}(a)}$ and property (6) follows.

For $s>1$ and $r \in(0,1]$, let

$$
\zeta(s, r)=\sum_{n=0}^{\infty} \frac{1}{(n+r)^{s}} .
$$

In particular, $\zeta(s, 1)=\zeta(s)$. These generalizations of the Riemann zeta function $\zeta(s)=\sum_{n=1}^{\infty} n^{-s}$ are very similar to those mentioned in [EMOT p. 24]. If $s=1$ and $r \in(0,1]$, define

$$
\zeta(1, r)=\sum_{n=0}^{\infty} \frac{1}{n+r}-\frac{1}{n+1} .
$$

The divergent series $\sum_{n} \frac{1}{n+r}$ is naturally identifiable with $\zeta(1, r)$ in the sense that whenever $\sum_{j=1}^{J} a_{j}=0$, the series $\sum_{n=1}^{\infty}\left(\sum_{j=1}^{J} a_{j} \frac{1}{n+r_{j}}\right)$ is convergent and equal to $\sum_{j} a_{j} \zeta\left(1, r_{j}\right)$. Notice that $O\left(n^{-1}\right)$ terms can appear in $r(n)$ only in this way, for otherwise $\sum r(n)$ would diverge.

Next, for $a \geq 2$ and $b$ integers, let

$$
\zeta_{a}(s)=\sum_{\substack{b=1 \\(b, a)=1}}^{a} \zeta\left(s, \frac{b}{a}\right) .
$$

\section{Lemma 1.}

(i) For $a$ and $s \geq 2$ positive integers, the number $\zeta_{a}(s)$ is an integer multiple of $\zeta(s)$. Explicitly, $\zeta_{a}(s)=\varphi_{s}(a) \zeta(s)$.

(ii) When $s=1$, the number $\zeta_{a}(1)$ is in the $\mathbb{Z}$-span of the natural logarithms of the prime divisors of a. Explicitly,

$$
\zeta_{a}(1)=\varphi(a) \sum_{i=1}^{r}\left(n_{i}+\frac{1}{p_{i}-1}\right) \log p_{i},
$$

where $a=p_{1}^{n_{1}} p_{2}^{n_{2}} \cdots p_{r}^{n_{r}}$.

Proof. For $s \geq 2$, we have

$$
a^{s} \zeta(s)=\sum_{b=1}^{a} \zeta\left(s, \frac{b}{a}\right)=\sum_{d \mid a} \sum_{\substack{b=1 \\(b, a)=d}}^{a} \zeta\left(s, \frac{b}{a}\right)=\sum_{d \mid a} \zeta_{\frac{a}{d}}(s)=\sum_{d \mid a} \zeta_{d}(s) .
$$


Therefore

$$
a^{s}=\sum_{d \mid a} \frac{\zeta_{d}(s)}{\zeta(s)}
$$

Apply the Möbius inversion formula and property (5) of Theorem 2 to get

$$
\frac{\zeta_{a}(s)}{\zeta(s)}=\sum_{d \mid a} \mu\left(\frac{a}{d}\right) d^{s}=\varphi_{s}(a) .
$$

When $s=1$, letting $\gamma_{n}=\sum_{k=1}^{n} \frac{1}{k}-\ln n$ be the defining sequence for Euler's constant, we calculate

$$
\begin{aligned}
\sum_{d \mid a} \zeta_{d}(1) & =\sum_{d \mid a} \zeta_{\frac{a}{d}}(1)=\sum_{d \mid a} \sum_{\substack{b=1 \\
(a, b)=d}}^{a} \lim _{n \rightarrow \infty} \sum_{k=0}^{n-1}\left(\frac{1}{k+\frac{b}{a}}-\frac{1}{k+1}\right) \\
& =\lim _{n \rightarrow \infty} \sum_{b=1}^{a} \sum_{k=0}^{n-1}\left(\frac{a}{a k+b}-\frac{1}{k+1}\right)=a \lim _{n \rightarrow \infty}\left(\sum_{k=1}^{a n} \frac{1}{k}-\sum_{k=1}^{n} \frac{1}{k}\right) \\
& =a \lim _{n \rightarrow \infty}\left(\gamma_{a n}+\ln (a n)-\gamma_{n}-\ln n\right)=a \ln a .
\end{aligned}
$$

By the Möbius inversion formula, we deduce that

$$
\begin{aligned}
\zeta_{a}(1)= & \sum_{d \mid a} \mu\left(\frac{a}{d}\right) d \ln d=a \ln a-\sum_{1 \leq i \leq r} \frac{a}{p_{i}} \ln \frac{a}{p_{i}}+\sum_{1 \leq i<j \leq r} \frac{a}{p_{i} p_{j}} \ln \frac{a}{p_{i} p_{j}}-\cdots \\
= & a \ln a-\sum_{1 \leq i \leq r} \frac{a}{p_{i}}\left(\ln a-\ln p_{i}\right)+\sum_{1 \leq i<j \leq r} \frac{a}{p_{i} p_{j}}\left(\ln a-\ln p_{i}-\ln p_{j}\right)-\cdots \\
= & a \ln a\left(1-\sum_{1 \leq i \leq r} \frac{1}{p_{i}}+\sum_{1 \leq i<j \leq r} \frac{1}{p_{i} p_{j}}-\cdots\right) \\
& +a \sum_{i=1}^{r}\left(1-\sum_{\substack{1 \leq j \leq r \\
j \neq i}} \frac{1}{p_{j}}+\sum_{\substack{1 \leq j<k \leq r \\
j, k \neq i}} \frac{1}{p_{i} p_{j}}-\cdots\right) \frac{\ln p_{i}}{p_{i}} .
\end{aligned}
$$

Now apply Property 3 of Theorem 2 ,

$$
\begin{aligned}
\zeta_{a}(1) & =\varphi(a) \ln a+\sum_{i=1}^{r} \frac{\varphi(a)}{1-\frac{1}{p_{i}}} \cdot \frac{\ln p_{i}}{p_{i}}=\varphi(a)\left(\ln a+\sum_{i=1}^{r} \frac{\ln p_{i}}{p_{i}-1}\right) \\
& =\varphi(a) \sum_{i=1}^{r}\left(n_{i}+\frac{1}{p_{i}-1}\right) \ln p_{i} .
\end{aligned}
$$

Here is some notation allowing $\zeta_{a}(1)$ to be written as a generic infinite sum of finite support. If $\mathcal{P}$ is the set of all prime numbers, $a>1$ is an integer, and $p \in \mathcal{P}$, we define $\exp _{a}(p)$ to be the exponent of $p$ in $a$, and the delta function $\delta_{a}(p)$ to be 1 , if $p \mid a$, and zero otherwise. Then we may write

$$
\zeta_{a}(1)=\varphi(a) \sum_{p \in \mathcal{P}}\left(\exp _{a}(p)+\frac{\delta_{a}(p)}{p-1}\right) \ln p .
$$


Lemma 2. Let $\mathcal{P}$ be the set of prime numbers. The set $\mathcal{A}=\{1\} \cup\{\ln p: p \in \mathcal{P}\}$ is linearly independent over $\mathbb{Q}$.

Proof. Suppose $\sum_{i=1}^{k} \alpha_{i} \ln p_{i}=q$, for some $\alpha_{i}$ and $q$ rational numbers. We may assume that the $\alpha_{i}$ and $q$ are actually integers by multiplying through by their common denominator. Exponentiation yields $\prod_{i=1}^{k} p_{i}^{\alpha_{i}}=e^{q}$. Since $e$ is transcendental, so is $e^{q}$, for any integer $q \neq 0$. Since $e^{q}$ is equal to an integer, we must have $q=0$. Finally, by the Fundamental Theorem of Arithmetic, all exponents $\alpha_{i}$ must be zero.

This theorem produces numerous previously unknown series with rational sums.

\section{Theorem 3.}

- A linear combination $\sum_{a \geq 2, s \text { even }} \beta_{a, s} \zeta_{a}(s)$ with rational coefficients $\beta_{a, s}$ is a rational number if and only if

$$
\sum_{a} \beta_{a, s} \varphi_{s}(a)=0, \text { for each even } s \geq 2 .
$$

- A linear combination $\sum_{a \geq 2} \beta_{a} \zeta_{a}(3)$ with rational coefficients $\beta_{a}$ is a rational number if and only if

$$
\sum_{a} \beta_{a} \varphi_{3}(a)=0
$$

- For each odd $s \geq 5$, a linear combination $\sum_{a \geq 2} \beta_{a} \zeta_{a}(s)$ with rational coefficients $\beta_{a}$ is a rational number if

$$
\sum_{a} \beta_{a} \varphi_{s}(a)=0
$$

- A linear combination $\sum_{a} \beta_{a} \zeta_{a}(1)$ with rational coefficients $\beta_{a}$ is a rational number if and only if

$$
\sum_{a} \beta_{a} \varphi_{s}(a)\left(\exp _{a}(p)+\frac{\delta_{a}(p)}{p-1}\right)=0
$$

for every prime number $p$.

Proof. All four statements are clear from Lemma 1. The first statement also needs Euler's formula showing that for even integers $s, \zeta(s)$ is a rational multiple of $\pi^{s}$, together with Lindemann's result that $\pi$ is transcendental. The second requires Apéry's result that $\zeta(3)$ is irrational $\mathrm{A}$. Notice that the third statement is only an implication since the irrationality of $\zeta(s)$ for odd $s \geq 5$ is unknown. The last statement is an immediate consequence of Lemma 2 and formula (3.2).

\section{Corollary 2.}

(i) $\operatorname{dim}_{\mathbb{Q}} \operatorname{span}_{\mathbb{Q}}\left\{\zeta_{a}(1): 2 \leq a \leq n\right\}=\pi(n)$, the number of primes $\leq n$.

(ii) $\operatorname{dim}_{\mathbb{Q}} \operatorname{span}_{\mathbb{Q}}\left\{\zeta_{a}(1): a \mid n\right\}=p(n)$, the number of prime divisors of $n$. 


\section{Conjectures}

Let $r(n)=P(n) / Q(n)$ be a rational form with integer coefficients with $\operatorname{deg} Q \geq$ $\operatorname{deg} P+2$. Our aim is to conjecture plausible and simple necessary and sufficient conditions on $P / Q$ for $\sum_{n=1}^{\infty} r(n)$ to be a rational number. By Theorem 1 , we may write

$$
\begin{aligned}
s & =\sum_{n=1}^{\infty} r(n)=\{\text { Telescoping part }\}+\{\text { Representative part }\} \\
& =\left\{\sum_{n=1}^{\infty}(R(n)-R(n+1))\right\}+\left\{\sum_{\substack { n=1 \\
\begin{subarray}{c}{f, s \\
\text { irred. } \\
\operatorname{deg} f \geq 2{ n = 1 \\
\begin{subarray} { c } { f , s \\
\text { irred. } \\
\operatorname { d e g } f \geq 2 } }\end{subarray}}^{\infty(n)^{s}}+\sum_{n=1}^{\infty} \sum_{\alpha \in(0,1] \cap \mathbb{Q}} \frac{a_{f s}}{(n+\alpha)^{s}}\right\} \\
& =\{I\}+\{I I+I I I\} .
\end{aligned}
$$

Of course, the first term $I$ immediately telescopes to the rational number $R(1)$. Our first conjecture is that $s$ is never rational when $I I \neq 0$. This is incorporated into Conjecture 3 below as condition (i). From now until the statement of Conjecture 3 we will operate under the assumption that $I I=0$, which is to say that the representative part of $Q$ splits over $Q$ and Corollary 1 prevails.

Here are three conjectures about the values of the zeta function:

$\zeta 1$ : The numbers $\zeta(2), \zeta(3), \zeta(4), \ldots$ are irrational,

$\zeta 2$ : the numbers $1, \zeta(2), \zeta(3), \zeta(4), \ldots$ are linearly independent over $\mathbb{Q}$,

$\zeta 3$ : the set $\{1\} \cup\{\ln p: p \in \mathcal{P}\} \cup\{\zeta(s): s \geq 2\}$ is linearly independent over $\mathbb{Q}$.

Also, here are three conjectures about infinite series with general term being a $\mathbb{Q}$-linear combination of generalized zeta functions:

$\zeta_{a} 1:$ Fix $s \geq 2$. Any linear combination $\sum_{a} \beta_{a, s} \zeta_{a}(s)$, with rational coefficients $\beta_{a, s}$, is a rational number precisely when $\sum_{a} \beta_{a, s} \varphi_{s}(a)=0$,

$\zeta_{a} 2$ : any linear combination $\sum_{a, s \geq 2} \beta_{a, s} \zeta_{a}(s)$, with rational coefficients $\beta_{a, s}$, is a rational number precisely when $\sum_{a} \beta_{a, s} \varphi_{s}(a)=0$, for each $s \geq 2$,

$\zeta_{a} 3$ : any linear combination $\sum_{a>1, s \geq 1} \beta_{a, s} \zeta_{a}(s)$, with rational coefficients $\beta_{a, s}$, is a rational number precisely when $\sum_{a} \beta_{a, s} \varphi_{s}(a)=0$, for each $s \geq 2$, and $\sum_{a} \beta_{a, 1} \varphi(a)\left(\exp _{a}(p)+\frac{\delta_{a}(p)}{p-1}\right)=0$, for all $p \in \mathcal{P}$.

Theorem 4. For $i=1,2,3$, statement $\zeta i$ is true if and only if statement $\zeta_{a} i$ is true.

Proof. If $i=1$, by Lemma 1(i), $\sum_{a} \beta_{a, s} \zeta_{a}(s)=\left(\sum_{a} \beta_{a, s} \varphi_{s}(a)\right) \zeta(s)$. If $i=2$, by Lemma 1(i) again, $\sum_{a, s>1} \beta_{a, s} \zeta_{a}(s)=\sum_{s>1}\left(\sum_{a} \beta_{a, s} \varphi_{s}(a)\right) \zeta(s)$. If $i=3$, by Lemma 1(ii) and Lemma 1(i)

$$
\begin{aligned}
\sum_{a>1, s \geq 1} \beta_{a, s} \zeta_{a}(s) & =\sum_{p}\left(\sum_{a} \beta_{a, 1} \varphi_{s}(a)\left(\exp _{a}(p)+\frac{\delta_{a}(p)}{p-1}\right)\right) \ln p \\
& +\sum_{s \geq 2}\left(\sum_{a} \beta_{a, s} \varphi_{s}(a)\right) \zeta(s)
\end{aligned}
$$


The first set of conjectures is obviously difficult, but it is reasonably plausible. Euler's formula showing that for even integers $n, \zeta(n)$ is a rational multiple of $\pi^{n}$, together with Lindemann's result that $\pi$ is transcendental imply that the set $S=\{1, \zeta(2), \zeta(4), \zeta(6), \ldots\}$ is independent over $\mathbb{Q}$. Furthermore, Apéry showed in $\mathrm{A}]$ that $\zeta(3)$ is irrational. So, evidence for conjecture $\zeta 1$ is provided by the known irrationality of all the elements of the set $\{\zeta(3), \zeta(2), \zeta(4), \zeta(6), \ldots\}$, evidence for conjecture $\zeta 2$ is provided by the $\mathbb{Q}$-independence of $S$, and evidence for conjecture $\zeta 3$ is provided both by the $\mathbb{Q}$-independence of $S$ and by Lemma 2

We next show how conjecture $\zeta_{a} 3$ sheds light on the question of the rationality of sums $\sum p(n) / q(n)$. Convergence forces $\operatorname{deg} q \geq \operatorname{deg} p+2$; suppose $q$ factors completely over $\mathbb{Q}$. Then $r(n)=p(n) / q(n)$ has a partial fractions decomposition

$$
r(n)=\sum_{s=1}^{\infty} \sum_{\mathcal{C} \in \mathbb{Q} / \mathbb{Z}} \sum_{b / a \in \mathcal{C}} \frac{C_{b / a, s}}{(n+b / a)^{s}}
$$

where all $C_{b / a, s}$ are rational. For each $\mathcal{C} \in \mathbb{Q} / \mathbb{Z}$, there is a unique irreducible rational fraction $b^{*} / a^{*} \in \mathcal{C} \cap(0,1]$. Recall that the standard form $r^{*}(n)$ of $r(n)$ is the rational form

$$
r^{*}(n)=\sum_{s=1}^{\infty} \sum_{\mathcal{C} \in \mathbb{Q} / \mathbb{Z}} \frac{\sum_{b / a \in \mathcal{C}} C_{b / a, s}}{\left(n+b^{*} / a^{*}\right)^{s}}
$$

Then

$$
r(n)-r^{*}(n)=\sum_{s=1}^{\infty} \sum_{\mathcal{C} \in \mathbb{Q} / \mathbb{Z}} \sum_{b / a \in \mathcal{C}} C_{b / a, s}\left[\frac{1}{(n+b / a)^{s}}-\frac{1}{\left(n+b^{*} / a^{*}\right)^{s}}\right] .
$$

By Section 1, the series $\sum_{n=1}^{\infty} r(n)-r^{*}(n)$ telescopes, hence it sums up to a rational number. In particular, $\sum_{n=1}^{\infty} r(n)$ is a rational number if and only if its standard form $\sum_{n=1}^{\infty} r^{*}(n)$ is a rational number. Another way to see that, when the denominator splits, passing from a series to its standard form does not affect rationality is that a finite integer shift of the variable changes the corresponding infinite sum by only a finite number of terms. For example, $\sum_{n=1}^{\infty} \frac{1}{(n+5 / 2)^{2}}=$ $\sum_{n=1}^{\infty} \frac{1}{(n+1 / 2)^{2}}-\frac{1}{(3 / 2)^{2}}-\frac{1}{(5 / 2)^{2}}$. Incidentally, by Corollary $1 \sum r(n)$ telescopes exactly when its standard form is 0 . Therefore, working with standard forms is equivalent to working modulo telescoping.

Now we can rephrase conjecture $\zeta_{a} 3$ as follows.

Conjecture 1. Let $r(n)=P(n) / Q(n)$ be a rational form with integer coefficients with $\operatorname{deg} Q \geq \operatorname{deg} P+2$. Suppose

(i) $Q(n)$ factors completely over the rationals, and

(ii) the standard form of $\sum r(n)$ is expressible as a $\mathbb{Q}$-linear combination of $\zeta_{a}(s)$ for some finite set of positive integers a and $s$, i.e., is of the form

$$
\sum_{a>1, s \geq 1} \beta_{a, s} \zeta_{a}(s)
$$

with all $\beta_{a, s} \in \mathbb{Q}$. 
A necessary and sufficient condition for $\sum r(n)$ to be a rational number is that

$$
\begin{aligned}
\sum_{a} \beta_{a, s} \varphi_{s}(a) & =0, \text { for all } s \geq 2 \text {, and } \\
\sum_{a} \beta_{a, 1} \varphi(a)\left(\exp _{a}(p)+\frac{\delta_{a}(p)}{p-1}\right) & =0, \text { for all prime numbers } p .
\end{aligned}
$$

We will make an even stronger conjecture by dropping the condition that only terms of the form $\zeta_{a}(s)$ appear in the standard form.

Conjecture 2. Let $r(n)=P(n) / Q(n)$ be a rational form with integer coefficients with $\operatorname{deg} Q \geq \operatorname{deg} P+2$. Suppose

(i) $Q(n)$ factors completely over the rationals.

$A$ necessary and sufficient condition for $\sum r(n)$ to be a rational number is that its standard form be expressible as a $\mathbb{Q}$-linear combination of $\zeta_{a}(s)$ for some finite set of positive integers $a$ and $s$, i.e., be of the form $\sum_{a>1, s \geq 1} \beta_{a, s} \zeta_{a}(s)$ with all $\beta_{a, s} \in \mathbb{Q}$ and that

$$
\begin{aligned}
\sum_{a} \beta_{a, s} \varphi_{s}(a) & =0, \text { for all } s \geq 2 \text {, and } \\
\sum_{a} \beta_{a, 1} \varphi(a)\left(\exp _{a}(p)+\frac{\delta_{a}(p)}{p-1}\right) & =0, \text { for all prime numbers } p .
\end{aligned}
$$

For this conjecture to be true we need even more independence than what was asserted in conjecture $\zeta 3$ above. We also need all the parts of each $\zeta_{a}(s)$ to be independent from one another. For example,

$$
\zeta_{3}(1)=\zeta(1,1 / 3)+\zeta(1,2 / 3)=3 \ln 3,
$$

but there are no further $\mathbb{Q}$-linear relations among the elements of the set

$$
\left\{\zeta_{3}(1), \zeta(1,1 / 3), \zeta(1,2 / 3)\right\}
$$

since $\zeta(1,1 / 3)=\frac{3}{2} \ln 3+\frac{\pi}{2 \sqrt{3}}$ and $\zeta(1,2 / 3)=\frac{3}{2} \ln 3-\frac{\pi}{2 \sqrt{3}}$. So what we need is for the elements of the set

$$
T=\{\zeta(s, b / a): s \geq 1, a \geq 1,(b, a)=1,1 \leq b \leq a)\} \cup\{1\}
$$

to be $\mathbb{Q}$-independent, except for dependencies induced by dependencies among the various $\zeta_{a}(s): s \geq 1, a \geq 1$. These arise from the definition of $\zeta_{a}(s)$ and the two parts of Lemma 1. A compact way to put this is to demand that the set

$\{1\} \cup\left\{\zeta\left(s, \frac{b}{a}\right): s \geq 1,1<b<a,(a, b)=1\right\} \cup\left\{\zeta\left(s, \frac{1}{2}\right): s>1\right\} \cup\left\{\zeta\left(1, \frac{1}{p}\right): p\right.$ prime $\}$

be a basis for the span of $T$ over $\mathbb{Q}$. In the light of conjecture $\zeta 3$, it follows that this conjecture is equivalent to asserting that the set

$$
\{1\} \cup\{\zeta(s, b / a): s \geq 1,1<b<a,(a, b)=1\} \cup\{\zeta(s): s>1\} \cup\{\ln p: p \text { prime }\}
$$

is a basis of the span of $T$.

Our final conjecture is even more bold but less supported. It basically says that all the ways that the sum of a series of rational forms can be rational have already been found. 
Conjecture 3. Let $r(n)=P(n) / Q(n)$ be a rational form with integer coefficients with $\operatorname{deg} q \geq \operatorname{deg} p+2$. A necessary and sufficient condition for $\sum r(n)$ to be rational is that the following three conditions hold:

(i) the representative part of $r(n)$ consists of only terms whose denominators split over the rationals, so that the representative part differs from the standard form by a rational number,

(ii) the standard form of $r(n)$ be expressible as a $\mathbb{Q}$-linear combination of $\zeta_{a}(s)$ for some finite set of positive integers $a$ and s, i.e., be of the form $\sum_{a>1, s \geq 1} \beta_{a, s} \zeta_{a}(s)$ with all $\beta_{a, s} \in \mathbb{Q}$, and

(iii) that

$$
\begin{aligned}
\sum_{a} \beta_{a, s} \varphi_{s}(a) & =0, \text { for all } s \geq 2 \text {, and } \\
\sum_{a} \beta_{a, 1} \varphi(a)\left(\exp _{a}(p)+\frac{\delta_{a}(p)}{p-1}\right) & =0, \text { for all prime numbers } p .
\end{aligned}
$$

We have looked at a number of examples wherein the representative part has terms involving irreducible polynomials of degree at least 2 , such as $\sum \frac{1}{n^{2}+a^{2}}$ with $a=1,2,3,4$, and have not found anything contrary. But since very few such series have been evaluated exactly, there really is not much additional evidence one way or the other for this conjecture.

\section{Motivation And $k$-TELESCOPing}

The motivation for this paper was a simple problem in a calculus textbook. Problem 34 of section 12.2 of James Stewart's Calculus, 4th ed., Brooks/Cole, 1999 , asks for the evaluation of

$$
S=\sum_{n=1}^{\infty} \frac{1}{n(n+1)(n+2)} .
$$

The straightforward solution

$$
\begin{aligned}
S & =\frac{1}{2} \sum_{n=1}^{\infty}\left(\frac{1}{n}-\frac{2}{n+1}+\frac{1}{n+2}\right) \\
& =\frac{1}{2} \sum_{n=1}^{\infty}\left(\frac{1}{n(n+1)}-\frac{1}{(n+1)(n+2)}\right)=\frac{1}{4}
\end{aligned}
$$

leads immediately to two different generalizations: the first is

$$
\frac{1}{n(n+1)(n+2) \cdots(n+k)}=\frac{1}{k !} \sum_{j=0}^{k}(-1)^{j}\left(\begin{array}{l}
k \\
j
\end{array}\right) \frac{1}{n+j},
$$

which Doron Zeilberger told us is a specialization of the Vandermonde-Chu relation since it can be reached by simple manipulation starting from $\sum_{j=0}^{k}\left(\begin{array}{c}n+k \\ k-j\end{array}\right)\left(\begin{array}{c}-n \\ j\end{array}\right)=\left(\begin{array}{c}k \\ k\end{array}\right)$ or ${ }_{2} F_{1}(-k, n ; n+1 ; 1)=\frac{(1)_{k}}{(n+1)_{k}}$ (see pp. $1-3$ of [GR] for the definitions of ${ }_{2} F_{1}$ 
and $(a)_{k}$ and for this identity); while the second uses the partial fraction fact $\frac{1}{n(n+k)}=\frac{1}{k}\left(\frac{1}{n}-\frac{1}{n+k}\right)$,

$$
\begin{aligned}
& \sum_{n=1}^{\infty} \frac{1}{n(n+1)(n+2) \cdots(n+k)} \\
& =\frac{1}{k} \sum_{n=1}^{\infty}\left(\frac{1}{n(n+1)(n+2) \cdots(n+k-1)}-\frac{1}{(n+1)(n+2) \cdots(n+k)}\right) \\
& =\frac{1}{k} \cdot \frac{1}{k !} .
\end{aligned}
$$

(This is well known, see, e.g., formula 5.1.24.7 of [PBM].)

More generally, the same argument that produced formula (5.2), when coupled with the application of $d$ telescopes, gives

$$
\sum_{n=1}^{\infty} \frac{1}{n(n+d) \cdots(n+k d)}=\frac{1}{k d} \sum_{\delta=1}^{d} \frac{1}{\delta(d+\delta) \cdots((k-1) d+\delta)} .
$$

When $a_{n} \rightarrow 0$, we have this basic telescoping formula

$$
\sum_{n=1}^{\infty}\left(a_{n}-a_{n+1}\right)=a_{1}
$$

It can easily be generalized to

$$
\sum_{n=1}^{\infty}\left(\sum_{\kappa=1}^{k} a_{n+\kappa-1} x_{\kappa}\right)=\sum_{\kappa=1}^{k-1} \sum_{j=0}^{k-1-\kappa} a_{\kappa+j} x_{\kappa},
$$

which we will call $k$-telescoping. An application of higher telescoping comes when we evaluate

$$
\sum_{n=1}^{\infty} \frac{1}{n(n+1)(n+2) \cdots(n+k)}
$$

in two different ways. On the one hand, the simple partial fraction argument given in formula (5.2) shows this sum to be $\frac{1}{k \cdot k !}$; on the other hand, applying formula (5.1) and then $k$-telescoping shows this sum to be $\frac{1}{k !} \sum_{\kappa=1}^{k} \sum_{j=0}^{k-\kappa}\left(\begin{array}{c}k \\ j\end{array}\right)(-1)^{j} \frac{1}{\kappa+j}$. Putting these two facts together gives the identity

$$
\sum_{\kappa=1}^{k} \sum_{j=0}^{k-\kappa}\left(\begin{array}{l}
k \\
j
\end{array}\right)(-1)^{j} \frac{1}{\kappa+j}=\frac{1}{k} .
$$

If we do exactly the same thing in the $q$ setting, starting from formulas (7.5) and (17.3) we arrive at a $q$-analogue of this identity, namely

$$
\sum_{\kappa=1}^{k} \sum_{j=0}^{k-\kappa}\left[\begin{array}{l}
k \\
j
\end{array}\right]_{q} q^{(j-1) j / 2}(-1)^{j} \frac{q^{\kappa+j}}{[\kappa+j]_{q}}=\frac{1}{[k]_{q}}\left(1-(1-q)^{k}[k]_{q} !\right) .
$$

There are also $q$ versions of the discrete integral mentioned above in connection with formula (1.2) [KC]. 


\section{Square roots, Cube roots, and Algebraic integers}

Generalizations of the basic square root example (1.6) are

$$
\sum_{n=1}^{\infty} \frac{1}{(\sqrt{n}+\sqrt{n+1})(\sqrt{n}+\sqrt{n+2})(\sqrt{n+1}+\sqrt{n+2})}=\frac{\sqrt{2}-1}{2},
$$

$$
\begin{aligned}
& \sum_{n=1}^{\infty} \frac{\sqrt{n}+\sqrt{n+1}+\sqrt{n+2}+\sqrt{n+3}}{(\sqrt{n}+\sqrt{n+1})(\sqrt{n}+\sqrt{n+2})(\sqrt{n}+\sqrt{n+3})(\sqrt{n+1}+\sqrt{n+2})(\sqrt{n+1}+\sqrt{n+3})(\sqrt{n+2}+\sqrt{n+3})} \\
& =\frac{-\sqrt{3}+2 \sqrt{2}-1}{6}=.016 \ldots,
\end{aligned}
$$

and, more generally,

$$
\begin{aligned}
\sum_{n=1}^{\infty} \frac{\phi_{k}(n)}{\prod_{0 \leq i<j \leq k}(\sqrt{n+i}+\sqrt{n+j})} & =\frac{1}{k !} \sum_{n=1}^{\infty}\left(\sum_{j=0}^{k}\left(\begin{array}{c}
k \\
j
\end{array}\right)(-1)^{k-j} \sqrt{n+j}\right) \\
& =\frac{1}{k !} \sum_{j=0}^{k-1}\left(\begin{array}{c}
k-1 \\
j
\end{array}\right)(-1)^{k-j} \sqrt{k-j}
\end{aligned}
$$

Here

$$
\begin{gathered}
\phi_{2}(n)=1, \phi_{3}(n)=s_{1}, \phi_{4}(n)=s_{1} s_{2}-s_{3} \\
\phi_{5}(n)=\left(9 s_{1}{ }^{4}-34 s_{1}{ }^{2} s_{2}+42 s_{1} s_{3}+14 s_{2}{ }^{2}-42 s_{4}-195\right) / 6
\end{gathered}
$$

where $s_{i}=s_{i}(k, n)$ is the $i$ th elementary symmetric function in the $k+1$ variables $\{\sqrt{n}, \ldots, \sqrt{n+k}\}$. For example, the $s_{2}$ appearing in the evaluation of $\phi_{4}(n)$ is $\sum_{0 \leq i<j \leq 4} \sqrt{n+i} \sqrt{n+j}$. Each of these formulas involves summing the underlying identity

$$
\frac{\phi_{k}(n)}{\prod_{0 \leq i<j \leq k}(\sqrt{n+i}+\sqrt{n+j})}=\frac{1}{k !} \sum_{j=0}^{k}\left(\begin{array}{l}
k \\
j
\end{array}\right)(-1)^{k-j} \sqrt{n+j}
$$

over all $n \geq 1$. We find these identities interesting because for each $k=1, \ldots, 5$, the left side is invariant under every permutation of the variables $\{\sqrt{n}, \ldots, \sqrt{n+k}\}$, while the right hand side is not. To be a little more explicit about this phenomenon, return to the identity underlying the basic square root example,

$$
\frac{1}{\sqrt{n}+\sqrt{n+1}}=\sqrt{n+1}-\sqrt{n}
$$

If the "variables" $\sqrt{n}$ and $\sqrt{n+1}$ are interchanged, the left side remains invariant, while the right side changes sign. This is possible because the quantities $\sqrt{n}$ and $\sqrt{n+1}$ are not independent. What we are trying to do is find a large class of expressions similar to $\sqrt{n+1}-\sqrt{n}$ which are easily summed by telescoping and which can be expressed as symmetric functions of the associated variables. Each time this is done, an example of a series with symmetrical terms that can be summed exactly is created.

We will present neither the derivations of formulas (6.1) and (6.2) nor the verification of formula (6.3) for the cases of $k=4$ and 5 , all of this being routine. It 
seems possible to guess a general formula from these five square root results, but we found the results (and also the derivations) becoming somewhat ponderous, so we will stop with the conjecture that for $k \geq 6, \phi_{k}$ is still a polynomial of degree $k-1$ in the variables $\{\sqrt{n}, \ldots, \sqrt{n+k}\}$ and $\phi_{k}$ can still be written as a polynomial in the elementary symmetric functions of those variables.

After square roots come cube roots. Here are two more formulas.

$$
\sum_{n=1}^{N} \frac{1}{(n+1)^{2 / 3}+(n+1)^{1 / 3} n^{1 / 3}+n^{2 / 3}}=\sum_{n=1}^{N} \sqrt[3]{n+1}-\sqrt[3]{n}=\sqrt[3]{N+1}-1 .
$$

For the second cube root formula, let $C(m, n)=m^{2 / 3}+m^{1 / 3} n^{1 / 3}+n^{2 / 3}$. Then

$$
\begin{aligned}
\sum_{n=1}^{\infty} \frac{\sqrt[3]{n}+\sqrt[3]{n+1}+\sqrt[3]{n+2}}{\prod_{0 \leq i<j \leq 2} C(n+i, n+j)} & =\frac{-1}{2} \sum_{n=1}^{\infty} \sqrt[3]{n}-2 \sqrt[3]{n+1}+\sqrt[3]{n+2} \\
& =\frac{-1}{2}(\sqrt[3]{1}-2 \sqrt[3]{2}+\sqrt[3]{2})=\frac{\sqrt[3]{2}-1}{2}
\end{aligned}
$$

There is an obvious generalization of equations (1.6) and (6.4) to $r$ th roots based on the identity

$$
1=(n+1)-1=\left((n+1)^{\frac{1}{r}}-n^{\frac{1}{r}}\right) \sum_{j=0}^{r-1} n^{\frac{j}{r}}(n+1)^{\frac{r-1-j}{r}} .
$$

For any natural numbers $r$ and $d$, let

$$
\Delta_{d, r}(n)=\frac{1}{d !} \sum_{j=0}^{d}\left(\begin{array}{l}
d \\
j
\end{array}\right)(-1)^{j} \sqrt[r]{n+j}
$$

denote the $d$ th forward difference of the $r$ th root of $n$. Then define $\phi_{r, d}(n)$, a polynomial in the $d+1$ variables $\sqrt[r]{n}, \sqrt[r]{n+1}, \ldots, \sqrt[r]{n+d}$, by

$$
\frac{\phi_{r, d}(n)}{\prod_{0 \leq i<j \leq k}(\sqrt[r]{n+i}+\sqrt[r]{n+j})}=\Delta_{d, r}(n) .
$$

A natural and fairly far-reaching generalization encompassing all the equations above is the conjecture that $\phi_{r, d}(n)$ can always be expressed as a symmetric polynomial in the variables $\sqrt[r]{n}, \sqrt[r]{n+1}, \ldots, \sqrt[r]{n+d}$. It is always possible to telescope $\sum_{n} \Delta_{d, r}(n)$, so a consequence of this conjecture is the existence of a lot of sums with symmetrical summands that can be computed exactly, namely one for every pair $(r, d)$.

One can hope to go even further than $k$ th roots, possibly all the way to algebraic numbers in general. Here is one small example that points in this direction. Let

$$
\sqrt{1+\sqrt{n}}=\alpha_{n}
$$

As above, $\sum_{n=1}^{N} \Delta \alpha_{n}=\alpha_{1}-\alpha_{N+1}$ provides motivation for wishing to find a representation of $\sqrt{1+\sqrt{n}}-\sqrt{1+\sqrt{n+1}}$ that is symmetric in the sense of being invariant under the transposition of the variables $\alpha_{n}$ and $\alpha_{n+1}$. We compute $\alpha_{n}^{2}=(\sqrt{1+\sqrt{n}})^{2}=1+\sqrt{n},\left(\alpha_{n}^{2}-1\right)^{2}=n, \alpha_{n}^{4}-2 \alpha_{n}^{2}+1=n$. Subtract this equation from $\alpha_{n+1}^{4}-2 \alpha_{n+1}^{2}+1=n+1$ and factor in order to obtain the 
equation $\left(\alpha_{n+1}-\alpha_{n}\right)\left[\left(\alpha_{n}+\alpha_{n+1}\right)\left(2-\alpha_{n}^{2}-\alpha_{n+1}^{2}\right)\right]=1$. The quantity in brackets is symmetric, so the desired relation is

$$
\alpha_{n+1}-\alpha_{n}=\frac{1}{\left(\alpha_{n}+\alpha_{n+1}\right)\left(2-\left(\alpha_{n}^{2}+\alpha_{n+1}^{2}\right)\right)} .
$$

\section{QUANTUM FORMULAS}

Fix a real number $q$. For each positive integer $n$, define $[n]_{q}=1+q+\cdots q^{n-1}=$ $\frac{q^{n}-1}{q-1}$. The idea of this section is to think of $q$ as a "generalization" of 1 and, consequently, of $[n]_{q}$ as a generalization of $n$. We will demand only that $|q|<1$, but we will informally think of $q$ as being close to 1 . A quantum formula for us is any true formula obtained essentially by replacing integers by their analogues in a standard formula that reduces back to that standard formula under the "formal" transformation $q \rightarrow 1$. To get a feeling for how these generalizations work, we will start by trying to get a $q$-analogue of the very first telescoping example (1.1). That example depended on the partial fraction fact that

$$
\frac{1}{n(n+1)}=\frac{1}{n}-\frac{1}{n+1} \text {. }
$$

The $q$ analogues of the two sides are $\frac{1}{[n]_{q}[n+1]_{q}}$ and $\frac{1}{[n]_{q}}-\frac{1}{[n+1]_{q}}=\frac{[n+1]_{q}-[n]_{q}}{[n]_{q}[n+1]_{q}}$. But $[n+1]_{q}-[n]_{q} \neq 1$, so a little ingenuity is called for. In fact,

$$
[n+1]_{q}-[n]_{q}=1+\cdots+q^{n}-\left(1+\cdots+q^{n-1}\right)=q^{n} .
$$

So one thing we might study is

$$
\sum_{n=1}^{\infty} \frac{q^{n}}{[n]_{q}[n+1]_{q}}
$$

Because of (7.1), it seems as though we have

$$
\sum_{n=1}^{\infty} \frac{q^{n}}{[n]_{q}[n+1]_{q}}=\sum_{n=1}^{\infty}\left(\frac{1}{[n]_{q}}-\frac{1}{[n+1]_{q}}\right)=\frac{1}{[1]_{q}}=1 .
$$

On the other hand, by equivalence, we have $\frac{1-q^{n}}{\left(\frac{1-q^{n}}{1-q}\right)}=\frac{1-q^{n+1}}{\left(\frac{1-q^{n+1}}{1-q}\right)}, \frac{1-q^{n}}{[n]_{q}}=\frac{1-q^{n+1}}{[n+1]_{q}}$, $\frac{1}{[n]_{q}}-\frac{q^{n}}{[n]_{q}}=\frac{1}{[n+1]_{q}}-\frac{q^{n+1}}{[n+1]_{q}}$, and

$$
\frac{1}{[n]_{q}}-\frac{1}{[n+1]_{q}}=\frac{q^{n}}{[n]_{q}}-\frac{q^{n+1}}{[n+1]_{q}} .
$$

Taking (17.2) into account and then telescoping gives this different, ostensibly contradictory, result

$$
1=\sum_{n=1}^{\infty}\left(\frac{1}{[n]_{q}}-\frac{1}{[n+1]_{q}}\right)=\sum_{n=1}^{\infty}\left(\frac{q^{n}}{[n]_{q}}-\frac{q^{n+1}}{[n+1]_{q}}\right)=\frac{q}{[1]_{q}}=q .
$$

What is wrong is the first calculation. When we write $\sum_{n=1}^{\infty}\left(a_{n}-a_{n+1}\right)=a_{1}$, this is shorthand for $\sum_{n=1}^{N-1}\left(a_{n}-a_{n+1}\right)=a_{1}-a_{N}$ and $\lim _{N \rightarrow \infty} a_{N}=0$. In the standard case, our shorthand worked because $\lim _{N \rightarrow \infty} \frac{1}{N}=0$, but here, as long as $|q|<1$, we have $\lim _{N \rightarrow \infty} \frac{1}{[N]_{q}}=\lim _{N \rightarrow \infty} \frac{1-q}{1-q^{N}}=1-q \neq 0$. Just to leave 
the reader no doubt, the conclusion of this introductory exploration of quantum telescoping is this identity: whenever $|q|<1$, we have

$$
\sum_{n=1}^{\infty} \frac{q^{n}}{[n]_{q}[n+1]_{q}}=q .
$$

This phenomenon also validates our choosing the $q$ analogue of $\sum_{n=1}^{\infty} \frac{1}{n(n+1)}$ to be $\sum_{n=1}^{\infty} \frac{q^{n}}{[n]_{q}[n+1]_{q}}$, rather than $\sum_{n=1}^{\infty} \frac{1}{[n]_{q}[n+1]_{q}}$, since the former is a convergent series, while the $n$th term of the latter tends to $(1-q)^{2}$, making the series strongly divergent.

Two more notational items are the quantum factorial,

$$
[k]_{q} != \begin{cases}1 & \text { if } k=0, \\ {[1]_{q} \cdots[k-1]_{q}[k]_{q}} & \text { if } k \geq 1,\end{cases}
$$

and, for integers $k$ and $j$, with $0 \leq j \leq k$, the quantum binomial coefficient,

$$
\left[\begin{array}{l}
k \\
j
\end{array}\right]_{q}=\frac{[k]_{q} !}{[j]_{q} ![k-j]_{q} !}
$$

We now find $q$ analogues of (5.1) and (5.2). For (5.1) we offer two generalizations. They are

$$
\frac{1}{[k]_{q} !} \sum_{j=0}^{k}\left[\begin{array}{c}
k \\
j
\end{array}\right]_{q} q^{(j-1) j / 2}(-1)^{j} \frac{q^{n+j}}{[n+j]_{q}}=\frac{q^{n}}{[n]_{q} \cdots[n+k]_{q}}
$$

and

$$
\frac{1}{[k]_{q} !} \sum_{j=0}^{k}\left[\begin{array}{l}
k \\
j
\end{array}\right]_{q} q^{(j-1) j / 2}(-1)^{j} \frac{1}{[n+j]_{q}}=\frac{q^{n}}{[n]_{q} \cdots[n+k]_{q}} .
$$

A satisfying generalization of relation (5.2) is that for $|q|<1$,

$$
\sum_{n=1}^{\infty} \frac{q^{n}}{[n]_{q} \cdots[n+k]_{q}}=\frac{1}{[k]_{q}}\left(\frac{1}{[k]_{q} !}-(1-q)^{k}\right) .
$$

We also give quantum versions of the standard factorial and square root formulas, (1.7) and (1.6). They are, for $|q|<1$,

$$
\sum_{n=1}^{\infty} \frac{q^{n}}{q^{n-1}[n-1]_{q} !+q^{n}[n]_{q} !}=\sum_{n=1}^{\infty} \frac{q}{[n-1]_{q} !+q[n]_{q} !}=\sum_{n=1}^{\infty} \frac{1}{[n]_{q} !}-\frac{1}{[n+1]_{q} !}=1
$$

and

$$
\sum_{n=1}^{N} \frac{q^{n}}{\sqrt{[n]_{q}}+\sqrt{[n+1]_{q}}}=\sqrt{[N+1]_{q}}-1
$$

We will not derive $q$ analogues of formulas (6.1) and (6.2). Doing so should not present any major difficulties.

After we had submitted this paper, R. Askey called our attention to the website of Ashish Sirohi where an understanding of some of the phenomena we investigate is displayed. 


\section{REFERENCES}

[A] Apéry, R., Interpolation de fractions continues et irrationalité de certaines constantes. (French) [Interpolation of continued fractions and irrationality of certain constants] Mathematics, pp. 37-53, CTHS: Bull. Sec. Sci., III, Bib. Nat., Paris, 1981. MR83b:10039

[EMOT] Erdélyi, A., Magnus, W., Oberhettinger, F., Tricomi, F. G., Higher Transcendental Functions. Vol. I. Based, in part, on notes left by Harry Bateman. McGraw-Hill, New York-Toronto-London, 1953. MR0058756 (15:419i)

[GR] Gasper, G. and Rahman, M., Basic Hypergeometric Series, Cambridge University Press, Cambridge, 1990. MR.1052153(91d:33034)

[HW] Hardy, G. H. and Wright, E. M., The Theory of Numbers, Oxford Univ. Press, Oxford, 1975. MR0568909 (81i:10002)

[KC] Kac, V. and Cheung, P., Quantum Calculus, Springer-Verlag, New York, 2001. MR.1865777 (2003i:39001)

[PBM] Prudnikov, A. P., Brychkov, Yu. A., and Marichev, O. I., Integrals and Series, Vol. 1, Gordon and Breach, Glasgow, 1986. MR0874986 (88f:00013)

Department of Mathematical Sciences, DePaul University, Chicago, Illinois 60614

E-mail address: mash@math.depaul.edu

URL: http://www.depaul.edu/ mash/

Department of Mathematical Sciences, DePaul University, Chicago, Illinois 60614

E-mail address: scatoiu@math.depaul.edu

URL: http://www. depaul.edu/ scatoiu/ 